\title{
Sleep in Children with Chronic Lung Disease
}

\author{
Refika Ersu
}

Marmara University, Istanbul, Turkey

S leep is a vulnerable period for the respiratory system. Patients with chronic lung disease (CLD) may be at risk for hypoxemia during sleep; they have reduced baseline arterial oxygen pressure that develops with advancing lung disease. Nocturnal hypercapnia may be present in CLD without nocturnal hypoxemia or daytime gas-exchange abnormalities. Increased upper airway resistance, including nasal obstruction, is a risk factor for obstructive sleep apnoea in children with cystic fibrosis and primary ciliary dyskinesia. Sleep problems are usually underestimated in these patients. Non-invasive ventilation and supplemental oxygen may be helpful in mitigating the adverse effects of nocturnal hypercapnia and hypoxemia. Sleep should be evaluated in children with CLD to improve the care of these patients.

DOl: https://doi.org/10.17925/ERPD.2017.03.01.21

\section{Keywords}

Childhood, chronic lung disease,

sleep-disordered breathing

Disclosure: Refika Ersu has no conflicts of interest to declare in relation to this article. This article is a short opinion piece and has not been submitted to external peer reviewers. No funding was received in the publication of this article.

Authorship: All named authors meet the International Committee of Medical Journal Editors (ICMJE) criteria for authorship of this manuscript, take responsibility for the integrity of the work as a whole, and have given final approval for the version to be published.

open Access: This article is published under the Creative Commons Attribution Noncommercial License, which permits any non-commercial use, distribution, adaptation and reproduction provided the original author(s) and source are given appropriate credit.

Received: 21 February 2017

Published Online: 24 July 2017

Citation: EU Respiratory \& Pulmonary Diseases, 2017;3(1):21-2

Corresponding Author: Refika Ersu, Marmara University, Division of Pediatric Pulmonology, Fevzi Çakmak Mah., Mimar Sinan Cd., No: 41 Üst Kaynarca, Pendik, Istanbul, Turkey. E: rersu@yahoo.com
As physicians, we evaluate patients while they are awake in our clinic. However, sleep is a vulnerable period for the respiratory system because of reductions in minute ventilation, lower lung volumes, increased upper airway resistance and positional ventilation-perfusion mismatching. ${ }^{1,2}$ Patients with chronic lung disease (CLD) may be at risk for hypoxemia during sleep; they have reduced baseline arterial oxygen pressure that develops over time with advancing lung disease. Also, ventilation-perfusion mismatching puts them closer to the steep-decline portion of the oxygen dissociation curve, so even the expected reduction in minute ventilation during sleep poses a risk for marked reductions in Sp02. Over time, progression of lung disease increases airway resistance and eventually results in alveolar hypoventilation. Sleep is normally characterised by hypoventilation related to decreased drive to the upper and lower respiratory muscles. Nocturnal hypercapnia may be present in CLD without nocturnal hypoxemia or daytime gas-exchange abnormalities. ${ }^{3-6}$

Increased upper airway resistance, including nasal obstruction, is a risk factor for obstructive sleep apnoea (OSA). Cystic fibrosis (CF) and primary ciliary dyskinesia (PCD) are associated with a high incidence of chronic sino-nasal disease, including nasal polyps. OSA is associated with considerable metabolic, cardiovascular and neurocognitive morbidity. Presence of OSA may put children with CLD at increased risk for growth failure and pulmonary hypertension. Patients with chronic lung disease experience both subjective and objective sleep disruption and gasexchange abnormalities during sleep, and sleep problems are usually underestimated in these patients. Non-invasive ventilation and supplemental oxygen may be helpful in mitigating the adverse effects of nocturnal hypercapnia and hypoxemia. ${ }^{6,7}$

More than $50 \%$ of CF patients have disturbed sleep, especially with advanced lung disease. They also have sleep complaints such as sleep-onset insomnia, frequent awakenings, night coughing, snoring and excessive daytime sleepiness. ${ }^{6}$ Even when stable, patients with $\mathrm{CF}$ report more frequent awakenings with coughing. When evaluated with objective methods, infants with CF have a sleep architecture similar to healthy controls. ${ }^{8}$ However, children with CF have lower sleep efficiency, reduced rapid eye movement (REM) sleep and increased electrocortical arousals. Children with more severe pulmonary disease (Iower forced expiratory volume in 1 second [FEV1]) have lower sleep efficiency and more nocturnal coughing. There is an association between decreased sleep efficiency and decreased mood profile, including happiness. ${ }^{6}$

Nocturnal hypoxemia in children with CF usually precedes diurnal hypoxemia and is generally unrecognised symptomatically. Significant hypoxemia is observed primarily during REM sleep and has been associated with pulmonary hypertension. Nocturnal hypoxemia is generally present when FEV1 is $<64 \%$ or if the baseline SpO2 is $<93-94 \%{ }^{6}$ In a study evaluating nocturnal hypoxia, $96 \%$ of children with CF, with normal pulmonary function tests or mild to moderate lung disease, had desaturation during sleep. Nocturnal oxygenation correlated with clinical and radiological scores, daytime $\mathrm{PaO} 2$ and $\mathrm{SaO} 2$, and Z-score of weight and height. ${ }^{9}$ 
When giving supplemental oxygen to patients with $\mathrm{CF}$, we need to balance the risks of developing pulmonary hypertension related to nocturnal hypoxemia against the costs, psychological impact, logistical difficulties, discomfort and potential for limiting mobility. Long-term oxygen treatment should be considered for hypoxic children with CF as a means to improve school attendance and for those who obtain symptomatic relief. In $\mathrm{CF}$, monitoring of $\mathrm{CO}_{2}$ levels should be carried out when oxygen therapy is initiated. ${ }^{10}$ Non-invasive ventilation, used in addition to oxygen, may improve gas exchange during sleep to a greater extent than oxygen therapy alone in moderate to severe disease. The benefits of noninvasive ventilation have largely been demonstrated in single treatment sessions with small numbers of participants. ${ }^{6}$ The impact of this therapy on pulmonary exacerbations and disease progression remains unclear.

OSA is very prevalent in children with CF. In a study of 63 children with CF, $56 \%$ had OSA syndrome (apnoea index $>1 / h$ ) and $26 \%$ had moderate to severe OSA (apnoea index $>5 / h$ ) OSA symptoms such as mouth breathing during sleep (83\%), difficulty breathing during sleep (70\%) and snoring greater than three times/week (38\%) were observed commonly among patients with CF.11

Children with non-CF bronchiectasis have chronic suppurative lung disease similar to CF. One study evaluating sleep in children with nonCF bronchiectasis showed that $37 \%$ of non-CF bronchiectasis patients compared to $17 \%$ of control children had poor sleep quality. ${ }^{12}$ Patients with sputum and wheezing had poorer sleep scores. The association of wheezing and breathlessness during night-time with sleep quality tended to be significant: $22 \%$ of non-CF bronchiectasis and $9 \%$ of controls had sleep disordered breathing. Non-CF bronchiectasis patients who snored had poorer sleep quality and patients with wheezing had significantly higher rates of snoring. Children with worse radiology scores also had worse sleep quality. ${ }^{12}$

Upper airway manifestations of PCD include chronic rhinosinusitis and nasal polyposis, which can increase upper airway resistance, which in turn can cause OSA. Some patients with PCD develop bronchiectasis with airflow obstruction and air trapping, which may lead to hypoxemia and hypercapnia. Abnormalities of lung mechanics and gas exchange may lead to sleep abnormalities similar to those seen in patients with CF and non-CF bronchiectasis. One study found that $65 \%$ of patients with PCD had habitual snoring. ${ }^{13}$ In polysomnography, $52 \%$ of patients with PCD had OSA. The OSA rate was higher in patients with PCD who snored. Habitual snoring and OSA were more common in patients with PCD who had cigarette smoke exposure in their homes..$^{13}$ Another study supported these findings: children with PCD had higher rates of obstructive apnoea compared with controls and lower oxygen saturation, which was associated with bronchiectasis severity score. ${ }^{14}$

Bronchiolitis obliterans (BO) is a rare form of chronic obstructive lung disease secondary to a severe insult to the lower respiratory tract that leads to a variable degree of inflammation and scarring, ultimately resulting in the narrowing and/or complete obliteration of the small airways. In children, the most common form is post-infectious BO, except in locations with a considerable number of paediatric lung or bone marrow transplant recipients. One study evaluating sleep in children with BO found that the risk of nocturnal hypoxia is increased in patients with post-infectious BO and is correlated with the severity of lung disease determined by pulmonary function tests. ${ }^{15}$ Although BO patients had short durations of central apnoea, they were more prone to be desaturated due to the apnoea. The decrease in SpO2 at the end of a six-minute walk test was an indicator of the risk of nocturnal hypoxia. ${ }^{15}$

In conclusion, patients with CLD experience both subjective and objective sleep disruption and gas-exchange abnormalities during sleep. Sleep problems are usually underestimated in these patients. Non-invasive ventilation and supplemental oxygen may be helpful in mitigating the adverse effects of nocturnal hypercapnia and hypoxemia. Sleep should be evaluated in children with CLD to improve the care of these patients. $\square$
1. Tabachnik E, Muller NL, Bryan AC, et al., Changes in ventilation and chest wall mechanics during sleep in normal adolescents, Appl Physiol Respir Environ Exerc Physiol, 1981;51:557-64.

2. Hudgel DW, Devadatta $P$, Decrease in functional residual capacity during sleep in normal humans, J Appl Physiol Respir Environ Exerc Physiol, 1984;57:1319-22.

3. Montgomery M, Wiebicke W, Bibi H, et al., Home measurement of oxygen saturation during sleep in patients with cystic fibrosis, Pediatr Pulmonol, 1989; 7:29-34.

4. Soni R, Dobbin CJ, Milross MA, et al., Gas exchange in stable patients with moderate-to-severe lung disease from cystic fibrosis, J Cyst Fibros, 2008;7:285-91.

5. Paiva R, Krivec U, Aubertin G, et al., Carbon dioxide monitoring during long-term noninvasive respiratory support in children, Intensive Care Med, 2009;35:1068-74.
Katz ES, Cystic fibrosis and sleep, Clin Chest Med 2014;35:495-504.

. Paranjape SM, MCGinley BM, Braun AT, Schneider H Polysomnographic markers in children with cystic fibrosis lung disease, Pediatrics, 2015;136:920-6.

8. Amin R, Bean J, Burklow K, Jeffries J, The relationship between sleep disturbance and pulmonary function in stable pediatric cystic fibrosis patients, CHEST, 2005;128:1357-63.

9. Uyan ZS, Ozdemir N, Ersu R, et al., Factors that correlate with sleep oxygenation in children with cystic fibrosis, Pediatr Pulmonol, 2007;42:716-22.

10. Balfour-Lynn IM, Field DJ, Gringras P, et al., BTS guidelines for home oxygen in children, Thorax, 2009;64 Suppl 2:ii1-26. 11. Ramos RT, Salles C, Gregório PB, et al., Evaluation of the upper airway in children and adolescents with cystic fibrosis and obstructive sleep apnea syndrome, Int J Pediat Otorhinolaryngol, 2009;73:1780-5.

12. Erdem E, Ersu R, Karadag B, et al., Effect of night symptoms and disease severity on subjective sleep quality in children with non-cystic-fibrosis bronchiectasis, Pediatr Pulmonol, 2011;46:919-26.

13. Oktem S, Karadag B, Erdem E, et al., Sleep disordered breathing in patients with primary ciliary dyskinesia, Pediatr Pulmonol, 2013;48:897-903

14. Santamaria F, Esposito M, Montella S, et al., Sleep disordered breathing and airway disease in primary ciliary dyskinesia, Respirology, 2014;19:570-5.

15. Uyan ZS, Turan I, Ay P, et al., Sleep disordered breathing and sleep quality in children with bronchiolitis obliterans, Pediatr Pulmonol, 2016;51:308-15. 